\title{
Metabolic engineering of Serratia marcescens MG1 for enhanced production of $(3 R)$-acetoin
}

Xin Lv' ${ }^{1}$ Lu Dai ${ }^{1}$, Fangmin Bai ${ }^{1}$, Zhanqing Wang ${ }^{1}$, Liaoyuan Zhang ${ }^{2^{*}}$ and Yaling Shen ${ }^{1 *}$

\begin{abstract}
Background: Optically pure acetoin (AC) is an important platform chemical which has been widely used to synthesize novel optically active a-hydroxyketone derivatives and liquid crystal composites.

Results: In this study, slaC and gldA encoding meso-2,3-butanediol dehydrogenase (meso-2,3-BDH) and glycerol dehydrogenase $(\mathrm{GDH})$, respectively, in S. marcescens MG1 were knocked out to block the conversion from $A C$ to 2,3-butanediol (2,3-BD). The resulting strain MG14 was found to produce a large amount of optically pure (3R)-AC with a little 2,3-BD, indicating that another enzyme responsible for 2,3-BD formation except meso-2,3-BDH and GDH existed in the strain MG1. Furthermore, SlaR protein, a transcriptional activator of AC cluster, was overexpressed using $P_{C}$ promoter in the strain MG14, leading to enhancement of the (3R)-AC yield by $29.91 \%$. The recombinant strain with overexpression of SlaR, designated as S. marcescens MG15, was used to perform medium optimization for improving (3R)-AC production.
\end{abstract}

Conclusion: Under the optimized conditions, $39.91 \pm 1.35 \mathrm{~g} / \mathrm{l}(3 R)$-AC was produced by strain MG15 with the productivity of $1.11 \mathrm{~g} / \mathrm{l} \mathrm{h}$ and the conversion rate of $80.13 \%$.

Keywords: (3R)-AC, Meso-2, 3-Butanediol dehydrogenase, Glycerol dehydrogenase, Transcriptional regulator, SlaR, Serratia marcescens MG1

\section{Background}

Acetoin (AC), also named as 3-hydroxy-2-butanone, is a widely used platform chemical which has been applied in many fields such as food additive, cosmetic products, pharmaceuticals, and chemical synthesis (Xiao and $\mathrm{Xu}$ 2007; Xiao and Lu 2014). (3R)-AC and (3S)-AC are two stereoisomeric forms of $\mathrm{AC}$, both of which are important pharmaceutical intermediates (Liu et al. 2011a). However, Chiral AC is usually more valuable, while it is often used to synthesize novel optically active $\alpha$-hydroxyketone derivatives and liquid crystal composites (Xiao et al. 2010).

\footnotetext{
*Correspondence: zliaoyuan@mail.ecust.edu.cn; ylshen@ecust.edu.cn 1 State Key Laboratory of Bioreactor Engineering, Shanghai Collaborative Innovation Center for Biomanufacturing Technology, East China University of Science and Technology, Shanghai 200237, China ${ }^{2}$ Key Laboratory of Biopesticide and Chemical Biology, Ministry of Education, College of Life Sciences, Fujian Agriculture and Forestry University, Fuzhou 350002, Fujian, China
}

A lot of microbials have been reported to be capable of producing a large amount of AC, such as Klebsiella pneumonia (Wang et al. 2015), Bacillus subtilis (Zhang et al. 2013a), Enterobacter cloacae (Zhang et al. 2016), and Paenibacillus polymyxa (Zhang et al. 2012). However, none of these strains had the ability to produce optically pure (3R)-AC or (3S)-AC. The current highest production of $75.2 \mathrm{~g} / \mathrm{l}$ AC was achieved from Serratia marcescens H32 without considering its isomer (Sun et al. 2012). Thus, $S$. marcescens was chosen to be the candidate to produce optically pure AC with high concentration in this study.

During the fermentation process, pyruvate produced from the glycolytic pathway was converted into $\alpha$-acetolactate by $\alpha$-acetolactate synthase ( $\alpha$-ALS) (Biswas et al. 2012). The majority of $\alpha$-acetolactate was subsequently transformed into (3R)-AC catalyzed by $\alpha$-acetolactate decarboxylase ( $\alpha$-ALDC), while a small amount of $\alpha$-acetolactate was transformed into diacetyl 
(DA), by the non-enzymatic oxidation decarboxylation (Liu et al. 2011b; Yang et al. 2014), and DA could be further converted into (3S)-AC which decreased the stereoisomeric purity of (3R)-AC. Both (3R)-AC and (3S)-AC were then transformed into 2,3-BD by the meso-2,3-BDH and the GDH encoded by slaC and gldA (Bai et al. 2015; Zhang et al. 2014), respectively, resulting low AC yield. So the native strain should be genetically modified for the production of optically pure AC.

It was found that the genes encoding $\alpha$-ALS and $\alpha$-ALDC usually formed a gene cluster, which was regulated by a transcriptional activator. The alsSD operon in Bacillus subtilis responsible for the expression of $\alpha$-ALS and $\alpha$-ALDC was regulated by the transcriptional regulator AlsR which was encoded by alsR (Renna et al. 1993; Frädrich et al. 2012), while the AC cluster in S.marcescens was found to be regulated by SlaR protein encoded by slaR (Rao et al. 2012). Since overexpression of AlsR had been proved to be successful for enhancing AC production by B.subtilis (Zhang et al. 2013b), similar protocol was attempted in S. marcescens MG1.

In this study, to obtain a $(3 R)$-AC producing strain, both the slaC and gldA were knocked out in S.marcescens MG1 using pUTKm1 as the suicide vector. The resulting mutant strain MG14 was found to produce single configuration of optically pure (3R)-AC from sucrose. Besides, three endogenous promoters were attempted to express the transcriptional regulator SlaR for the purpose of enhancing the production of (3R)-AC. Finally, the optimal culture conditions and the suitable fermentation medium component were developed for the engineered strain using statistical methods.

\section{Methods}

\section{Enzymes and chemicals}

Restriction enzymes were purchased from Thermo Fisher (USA). T4 DNA ligase and Primestar were purchased from TaKaRa Biotech (Dalian, China). Taq DNA polymerase was purchased from CW Bio (Beijing, China). Bacterial DNA kit was purchased from SBS Genetech (Beijing, China), plasmid mini kit, cycle-pure kit, and gel extraction kit were purchased from Omega Biotech (Norcross, America). AC and 2,3-BD were purchased from Sigma-Aldrich (Shanghai, China).

\section{Constructing of S.marcescens $\Delta$ slaC, S.marcescens $\Delta g / d \mathrm{~A}$, and S.marcescens $\Delta$ slaC $\Delta$ gldA}

The strains, plasmids, and primers used in this study are listed in Table 1. The suicide plasmid pUTKm1 (Lorenzo et al. 1990) was used to knock out slaC and gldA in Serrata marcescens MG1. In the case of slaC, the left and right flanking sequences of sla $\mathrm{C}$ were amplified from the genomic DNA of S.marcescens MG1 and then fused by overlap PCR. The $\Delta s l a \mathrm{C}$ fragments were gel purified and then ligated into pUTKm1. The resulting plasmid was designated pUT-slaC. For conjugation, E. coli S17-1 $\lambda$ pir was used as the donor strain to amplify and transfer the suicide plasmid pUT-slaC. The conjugation cells were recovered with fresh LB and plated on selection medium plates. One of the kanamycin-resistant transconjugants (single-crossover) was picked on LB plate containing $50 \mu \mathrm{g} / \mathrm{ml}$ kanamycin. The single-crossover was then inoculated at $30{ }^{\circ} \mathrm{C}$ for $12 \mathrm{~h}$ on LB plate without antibiotic; Kanamycin-sensitive colonies from a double crossover event were verified by PCR using primers slaC-F1 and slaC-R2 to obtain the slaC mutant strain MG12. The gldA from MG1 and MG12 was knocked out individually in the same way to construct the gldA mutant strain MG13 and the double-knockout strain MG14.

\section{Expression of slaR with different promoters in engineered strain}

The construction of recombinant plasmids is also shown in Table 1. The sla $\mathrm{R}$ gene encoding the transcriptional regulator SlaR was amplified from the genomic DNA of S.marcescens MG1 using primers slaR-1 and slaR-2. The expression plasmid PAC-slaR was constructed by inserting gene slaR into pACYC184 (Sun et al. 2015) between the HindIII and BamHI sites. The cassettes of promoter $P_{\mathrm{C}}$ (promoter of slaC), $P_{\mathrm{A}}$ (promoter of slaA), and $P_{\mathrm{R}}$ (promoter of slaR) were amplified from the genomic DNA of MG1 using primer pairs $P_{\mathrm{C}^{-}} 1 / P_{\mathrm{C}^{-}}, P_{\mathrm{A}}-1 / P_{\mathrm{A}}-2$, and $P_{\mathrm{R}}-1 / P_{\mathrm{R}}-2$. The three purified PCR products were

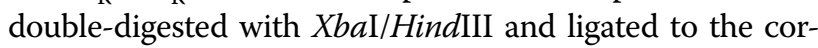
responding sites of $\mathrm{pAC}$-slaR, respectively, to generate pAC- $P_{\mathrm{C}}-$ slaR, pAC- $P_{\mathrm{AB}}$-slaR, and pAC- $P_{\mathrm{R}}$-slaR. The vectors were then introduced into $S$. marcescens MG14 separately by electroporation. The recombinants were selected on LB plates supplemented with $25 \mu \mathrm{g} / \mathrm{ml}$ chloramphenicol and designated as MG15, MG16, and MG17, respectively.

\section{Enzyme activity assays}

The engineered strains were grown at $30 \mathrm{C}$ for $10 \mathrm{~h}$, and then the fermentation broth was centrifuged at $8000 \mathrm{rpm}$ for $10 \mathrm{~min}$. After washing twice and resuspending with $50 \mathrm{mM}$ phosphate buffer $(\mathrm{pH} 7.0)$, the cells were disrupted with an ultrasonic cell breaking apparatus (Xinzhi, Ningbo, China). The resulting supernatant was finally obtained through centrifugation and was used for assaying enzyme activities (Kousoulos et al. 2006).

The AC reductase (AR) activities were determined by measuring NADH oxidation at $340 \mathrm{~nm}$ using a UV/visible spectrophotometer (UV-7504, Xin Mao, China). (Wayne 2008; Hao et al. 2014). The reaction was initiated by adding $20 \mu \mathrm{l}$ crude extract to $1 \mathrm{ml}$ reaction buffer which contained $67 \mathrm{mM}$ phosphate buffer (pH 7.4), $5 \mathrm{mM}$ of 
Table 1 Strains, plasmids, and primers used in this study

\begin{tabular}{|c|c|c|}
\hline Strains, plasmids and primers & Description & Source \\
\hline E.colis17-1 $(\lambda$ pir $)$ & recA thi pro $h s d R^{-} M^{+} R P 4:: 2-T c:: M u:: K m$ Tn7 lysogenized with $\lambda$ pir phage & Laboratory stock \\
\hline S. marcescens MG1 & Wild type, $T c^{r} A p^{r}$ & Laboratory stock \\
\hline MG12 & S. marcescens MG1 $\triangle$ slaC & This study \\
\hline MG13 & S. marcescens MG1 $\Delta g / d \mathrm{~A}$ & This study \\
\hline MG14 & S. marcescens MG1 $\Delta$ slaC $\Delta g / d \mathrm{~A}$ & This study \\
\hline MG15 & S. marcescens MG1 $\triangle$ slaC $\Delta g / d \mathrm{~A} / \mathrm{pACP} \mathrm{C}^{-}$slaR & This study \\
\hline MG16 & S. marcescens MG1 $\triangle$ slaC $\Delta g / d \mathrm{~A} / \mathrm{pACP}_{\mathrm{AB}}-\mathrm{s} / \mathrm{aR}$ & This study \\
\hline MG17 & S. marcescens MG1 $\triangle$ slaC $\Delta g / d \mathrm{~A} / \mathrm{pACP} \mathrm{R}_{\mathrm{R}}-\mathrm{s} / \mathrm{aR}$ & This study \\
\hline pUTKm1 & $\mathrm{Ap}^{r} \mathrm{Km}^{\mathrm{r}}$ oriR6 K oriTRP4 & (Lorenzo et al. 1990) \\
\hline pUT-slaC & pUTKm1 containing a 1798 bp deletion of slaC & This study \\
\hline pUT-g/dA & pUTKm1 containing a 1804 bp deletion of gldA & This study \\
\hline pACYC184 & $\mathrm{Cm}^{\mathrm{R}}$ & (Sun et al. 2015) \\
\hline pACP $C^{-s / a R}$ & pACYC184 containing slaR under the promotor of slaC & This study \\
\hline $\mathrm{pACP}_{\mathrm{A}^{-}-\mathrm{slaR}}$ & pACYC184 containing slaR under the promotor of slaA & This study \\
\hline $\mathrm{pACP} \mathrm{R}_{-}-\mathrm{SlaR}$ & pACYC184 containing slaR under the promotor of slaR & This study \\
\hline slaC-F1 & GTggtaccCATGCGGCAAGGAGCGCCATC & This study \\
\hline slaC-F2 & GGCCTGTGCGTTAACGCGAGACCTCCTCCATGTGAAC & This study \\
\hline slaC-R1 & GTTCACATGGAGGAGGTCTCGCGTTAACGCACAGGCC & This study \\
\hline slaC-R2 & GTgagtactCAGCCGCATCAGCCGCTAC & This study \\
\hline gldA-F1 & TTCggtaccGGTTGCGTTCAATGATGATG & This study \\
\hline gldA-F2 & CTCCCTACAAGGATCCGGTTTACCCTTGGGGCGCGGTGTGC & This study \\
\hline gldA-R1 & GCACACCGCGCCCCAAGGGTAAACCGGATCCTTGTAGGGAG & This study \\
\hline gldA-R2 & GCTagatctCTGCATGCTGGTCTGCTTGG & This study \\
\hline$P_{C^{-1}}$ & GTtctagaTCGCGGCCGCCTGCGGGC & This study \\
\hline$P_{C^{-2}}$ & GTaagcttGAGACCTCCTCCATGTG & This study \\
\hline$P_{A^{-1}}$ & GCtctagaAAAACGTAATATACGTTT & This study \\
\hline$P_{A^{-1}}$ & GCaagcttCTGACTGAGGAGGTGGTC & This study \\
\hline$P_{R^{-1}}$ & GCtctagaCTGACTGAGGAGGTGGTCGC & This study \\
\hline$P_{\mathrm{R}}-2$ & GCaagcttTTTTGCATTATATGCAAA & This study \\
\hline slaR-1 & GCaagcttATGAATGACGCACGCTATG & This study \\
\hline slaR-2 & GCggatccAATAGGGGTCGACCCGCCAA & This study \\
\hline
\end{tabular}

$\mathrm{AC}$, and $0.2 \mathrm{mM}$ of NADH. One unit of AR activity was defined as the amount of enzyme that consumed $1 \mu \mathrm{M}$ of $\mathrm{NADH}$ per min at $30 \mathrm{C}$.

To determine the integrated activities of $\alpha$-ALS and $\alpha$-ALDC, the reaction was initiated by addition of $100 \mu \mathrm{l}$ of crude extract to $3 \mathrm{ml}$ reaction buffer containing $100 \mathrm{mM}$ potassium phosphate buffer ( $\mathrm{pH}$ 6.5), $20 \mathrm{mM}$ sodium pyruvate, $0.01 \mathrm{mM}$ flavin adenine dinucleotide (FAD), $0.5 \mathrm{mM}$ thiamine pyrophosphate (TPP), and $0.5 \mathrm{mM} \mathrm{MgCl}_{2}$, and then the reaction was terminated by adding $0.5 \mathrm{ml} 2 \mathrm{M} \mathrm{NaOH}$. One unit of the integrated activity of $\alpha$-ALS and $\alpha$-ALDC was defined as the amount of enzyme required for the formation of $1 \mu \mathrm{M}$ AC per milligram protein per minute at $30{ }^{\circ} \mathrm{C}$. Total protein concentrations were determined using the Bradford method described by Marion (1976).

\section{Analytical methods}

The cell density was monitored by determining the absorbance at $600 \mathrm{~nm}$ using a spectrophotometer (UV-2008 h, Unic). To determine the residual sucrose concentrations of the samples, the sucrose of the samples was hydrolyzed to glucose by $2 \mathrm{M} \mathrm{H}_{2} \mathrm{SO}_{4}$ and determined using a bioanalyzer (SBA-40D, Shandong Academy of Sciences, China) after centrifugation. The intracellular $\mathrm{NADH}$ and $\mathrm{NAD}^{+}$concentrations were determined by procedures described in the previous studies (Snoep et al. 1991). Extracellular metabolites were measured using an HPLC (Agilent 1100) system equipped with an SB-Aq C18 $(4.6 \mathrm{~mm} \times 250 \mathrm{~mm})$ column. The column temperature was $30{ }^{\circ} \mathrm{C}$, and the mobile phase was $0.01 \mathrm{M} \mathrm{KH}_{2} \mathrm{PO}_{4}(\mathrm{pH}=2.12)$ with the flow rate at $0.6 \mathrm{ml} / \mathrm{min}$. $\mathrm{AC}$ and $2,3-\mathrm{BD}$ in the 
fermentation broth were extracted by ethyl acetate with the addition of isoamyl alcohol as internal standard and then quantified by GC (Agilent GC9860) equipped with a chiral column (FID-detector, Supelco $\beta$-DEX ${ }^{\mathrm{TM}} 120$, $30 \mathrm{~m}$ length, $0.25 \mathrm{~mm}$ inner diameter). The operation conditions used were as follows: $N_{2}$ was used as the carrier gas at a flow rate of $1.2 \mathrm{ml} / \mathrm{min}$; the injector temperature and the detector temperature were 215 and $245{ }^{\circ} \mathrm{C}$, respectively; the oven temperature was maintained at $50{ }^{\circ} \mathrm{C}$ for $1.5 \mathrm{~min}$, and then raised to $180{ }^{\circ} \mathrm{C}$ at a rate of $8{ }^{\circ} \mathrm{C} / \mathrm{min}$. A calibration curve was used to determine the concentration of the products.

\section{Medium and growth conditions}

Luria-Bertani (LB) medium of $50 \mathrm{ml}$ in $250 \mathrm{ml}$ flask was used for culturing E. coli, S. marcescens MG1, and its derivatives on a rotary shaker at $200 \mathrm{rpm}$. E. coli was grown at $37{ }^{\circ} \mathrm{C}$ and $S$. marcescens MG1 was grown at $30{ }^{\circ} \mathrm{C}$. If necessary, the LB medium was supplemented with $100 \mu \mathrm{g} / \mathrm{ml}$ ampicillin, $50 \mu \mathrm{g} / \mathrm{ml}$ kanamycin, or $25 \mu \mathrm{g} /$ $\mathrm{ml}$ chloramphenicol.

For fermentation experiments, seeds cultivation was conducted in $250 \mathrm{ml}$ shake flasks containing $30 \mathrm{ml}$ fresh seed culture for $12 \mathrm{~h}$ and then inoculated $(5 \%, \mathrm{v} / \mathrm{v})$ into a $250 \mathrm{ml}$ shake flask with $50 \mathrm{ml}$ of fermentation medium at $30^{\circ} \mathrm{C}$ on a rotary shaker at $200 \mathrm{rpm}$. The seed medium was composed of the following $(\mathrm{g} / \mathrm{l})$ : glucose 10 , peptone 2 , yeast extract $1,\left(\mathrm{NH}_{4}\right)_{2} \mathrm{SO}_{4} 6, \mathrm{KH}_{2} \mathrm{PO}_{4} 10, \mathrm{NaCl} 0.5$, and $\mathrm{MgSO}_{4} 0.5$ at $\mathrm{pH}$ 7.2. The initial fermentation medium was composed of the following $(\mathrm{g} / \mathrm{l})$ : sucrose 90 , yeast extract 25 , sodium citrate 14 , sodium acetate $4, \mathrm{NH}_{4} \mathrm{H}_{2} \mathrm{PO}_{4} 3$, $\mathrm{MgSO}_{4}$ 0.5, and $\mathrm{MnSO}_{4} 0.05$ (Rao et al. 2012).

\section{Optimization of culture conditions and medium composition}

For culture conditions, the effects of temperature $(28,30$, $\left.33,35,37^{\circ} \mathrm{C}\right), \mathrm{pH}(6.0,6.5,7,7.5$, and 8.0), liquid volume $(10,20,30,40$, and $50 \mathrm{ml})$, and inoculation size $(1,3,5$, 7 , and $9 \%, \mathrm{v} / \mathrm{v})$ on (3R)-AC production on engineered $S$. marcescens were investigated. The whole fermentation process was conducted for $36 \mathrm{~h}$. The optimum culture conditions were chosen for experiments of medium optimization.

For medium optimization, sucrose, yeast extract, sodium citrate, sodium acetate, $\mathrm{NH}_{4} \mathrm{H}_{2} \mathrm{PO}_{4}, \mathrm{MgSO}_{4}$, and $\mathrm{MnSO}_{4}$ were chosen for further optimization based on our preliminary experiments. Plackett-Burman (PB) design was used to select factors that significantly influenced (3R)-AC production. Table 2 lists the levels of the seven variables and Table 3 shows the design details and responsible results. Variables with significant effects on (3R)-AC production were screened from $\mathrm{PB}$ design for further optimization. Response surface methodology (RSM) based on central composite design (CCD) was conducted to determine the optimal levels of the variables with significant effects for improving (3R)-AC production. The design details are given in Table 4. The experimental designs and analysis were conducted with Minitab 15.0 (Minitab Inc., State College, PA, USA). All experiments were repeated three times.

\section{Results and discussion}

Growth and metabolic profiles of S. marcescens MG1 and the mutant trains

Serratia marcescens was regarded to be a good 2,3-BDproducing strain. As shown in the study of Rao et al. (2012), S. marcescens MG1 produced 42.5 g/l 2,3-BD under $24 \mathrm{~h}$ of flask fermentation using sucrose as the substrate, while only $4.6 \mathrm{~g} / \mathrm{l}$ of $\mathrm{AC}$ was accumulated during the fermentation process. As the precursor of 2,3-BD, $\mathrm{AC}$ was readily converted into $2,3-\mathrm{BD}$, resulting in $\mathrm{AC}$ production with low yield. Two enzymes, meso-2,3-BDH and GDH, respectively, encoded by slaC and gldA were found to be responsible for the reduction of $\mathrm{AC}$ to 2,3$\mathrm{BD}$. Three mutant strains were constructed by knocking out the slaC and gldA individually or in combination. Fermentation experiments were subsequently conducted to investigate the metabolic profiles of the strains.

As shown in Fig. 1 and Table 6, deletion of slaC or/ and gldA reduced the AR activity and improved the

Table 2 The Plackett-Burman design for screening variables in (3R)-AC production

\begin{tabular}{llllrrrr}
\hline Factors $(\mathbf{g} / \mathbf{l})$ & Variables & Low level $(\mathbf{- 1})$ & High level $(+\mathbf{1})$ & Effect & Coef. & $\boldsymbol{t}$ value & $\boldsymbol{p}$ value \\
\hline Sucrose & $X_{1}$ & 60 & 90 & 3.310 & 0.322 & 10.29 & 0.001 \\
Yeast extract & $X_{2}$ & 15 & 25 & 0.287 & 0.322 & 0.89 & 0.423 \\
Sodium citrate & $X_{3}$ & 5 & 15 & 0.602 & 0.322 & 1.87 \\
Sodium acetate & $X_{4}$ & 1 & 3 & -1.012 & 0.322 & -3.14 & 0.135 \\
$\mathrm{NH}_{4} \mathrm{H}_{2} \mathrm{PO}_{4}$ & $X_{5}$ & 0.2 & 0.5 & 0.628 & 0.322 & 1.95 \\
$\mathrm{MgSO}_{4}$ & $X_{6}$ & 0.2 & 0.5 & -0.057 & 0.322 & -0.18 & 0.123 \\
$\mathrm{MnSO}_{4}$ & $X_{7}$ & 0.02 & 0.05 & -0.842 & 0.322 & -2.62 & 0.059 \\
\hline
\end{tabular}

$R^{2}=97.03 \%, R^{2}($ adj $)=91.83 \%$

Coef coefficient 
Table 3 The Plackett-Burman design variables (in coded levels) with (3R)-AC as response

\begin{tabular}{|c|c|c|c|c|c|c|c|c|}
\hline \multirow[t]{2}{*}{ Run } & \multicolumn{7}{|c|}{ Variable levels } & \multirow[t]{2}{*}{$(3 R)-\mathrm{AC}(\mathrm{g} / \mathrm{l})$} \\
\hline & $x_{1}$ & $x_{2}$ & $x_{3}$ & $X_{4}$ & $x_{5}$ & $x_{6}$ & $x_{7}$ & \\
\hline 1 & -1 & -1 & 1 & 1 & 1 & -1 & 1 & 20.68 \\
\hline 2 & -1 & -1 & -1 & 1 & 1 & 1 & -1 & 20.67 \\
\hline 3 & -1 & 1 & -1 & -1 & -1 & 1 & 1 & 19.78 \\
\hline 4 & 1 & -1 & 1 & 1 & -1 & 1 & -1 & 26.76 \\
\hline 5 & 1 & -1 & -1 & -1 & 1 & 1 & 1 & 27.78 \\
\hline 6 & 1 & -1 & 1 & -1 & -1 & -1 & 1 & 27.69 \\
\hline 7 & 1 & 1 & -1 & 1 & 1 & -1 & 1 & 26.66 \\
\hline 8 & 1 & 1 & -1 & 1 & -1 & -1 & -1 & 26.80 \\
\hline 9 & -1 & 1 & 1 & -1 & 1 & -1 & -1 & 23.81 \\
\hline 10 & -1 & -1 & -1 & -1 & -1 & -1 & -1 & 22.84 \\
\hline 11 & 1 & 1 & 1 & -1 & 1 & 1 & -1 & 32.31 \\
\hline 12 & -1 & 1 & 1 & 1 & -1 & 1 & 1 & 20.50 \\
\hline
\end{tabular}

Table 4 The design and results based on CCD

\begin{tabular}{|c|c|c|c|c|c|}
\hline \multirow[t]{2}{*}{ Run } & \multicolumn{2}{|l|}{ Sucrose } & \multicolumn{2}{|c|}{ Sodium acetate } & \multirow[t]{2}{*}{$(3 R)-\mathrm{AC}(\mathrm{g} / \mathrm{l})$} \\
\hline & Code $X_{1}$ & $X_{1}(\mathrm{~g} / \mathrm{l})$ & Code $X_{2}$ & $X_{2}(\mathrm{~g} / \mathrm{l})$ & \\
\hline 1 & 0 & 80.00 & 0 & 2.00 & 33.23 \\
\hline 2 & 0 & 80.00 & 0 & 2.00 & 33.79 \\
\hline 3 & 1 & 100.00 & 1 & 3.00 & 34.43 \\
\hline 4 & 1.41421 & 108.284 & 0 & 2.00 & 38.89 \\
\hline 5 & -1 & 60.00 & 1 & 3.00 & 24.96 \\
\hline 6 & 0 & 80.00 & -1.41421 & 0.58579 & 34.2 \\
\hline 7 & -1.41421 & 51.716 & 0 & 2.00 & 25.06 \\
\hline 8 & 0 & 80.00 & 1.41421 & 3.41421 & 31.06 \\
\hline 9 & 0 & 80.00 & 0 & 2.00 & 34.83 \\
\hline 10 & 1 & 100.00 & -1 & 1.00 & 39.48 \\
\hline 11 & -1 & 60.00 & -1 & 1.00 & 29.3 \\
\hline 12 & 0 & 80.00 & 0 & 2.00 & 34.51 \\
\hline 13 & 0 & 80.00 & 0 & 2.00 & 33.84 \\
\hline
\end{tabular}

AC production. (3R)-AC concentrations of 19.79 and $6.52 \mathrm{~g} / \mathrm{l}$ were obtained from strains MG 12 and MG 13, respectively, while only $2.92 \mathrm{~g} / \mathrm{l}(3 R)$ - AC was produced from $S$. marcescens MG1 (Table 6). This result demonstrated that the inactivation of slaC or gldA had positive effects on (3R)-AC production. However, lower cell density, longer fermentation time, and lower comprehensive titer of $\mathrm{AC}$ and 2,3-BD were observed after slaC inactivation. The slaC and gldA double-knockout strain MG 14 accumulated the highest concentration of (3R)-AC without (3S)-AC. This indicated that slaC and gldA were the only two genes responsible for the production of (3S)-AC in S. marcescens MG1. Therefore, S. marcescens MG14 was chosen for further investigation for (3R)-AC production.

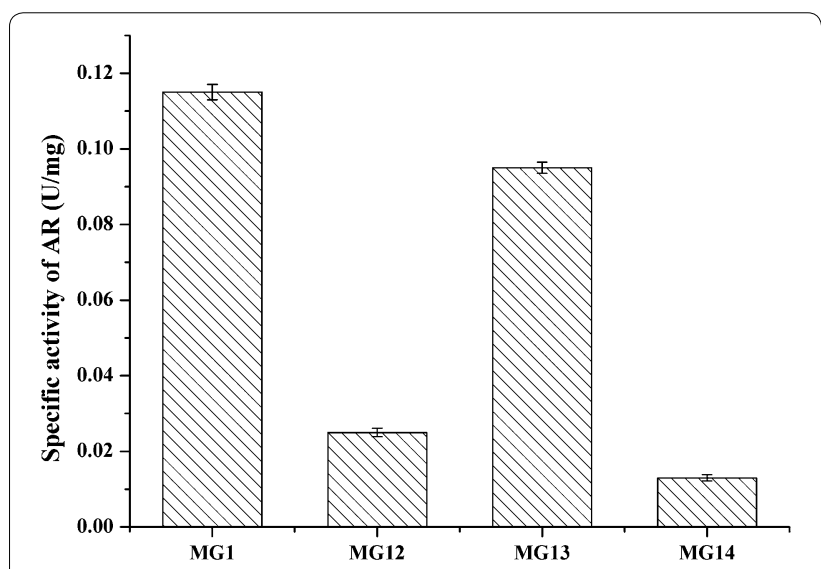

Fig. 1 The enzyme activity of AR of MG1, MG12, MG13, and MG14

In addition to $\mathrm{AC}$ production, the intracellular $\mathrm{NADH}$ and $\mathrm{NAD}^{+}$concentration was also observed. Table 5 shows that disruption of slaC and/or gldA could increase the $\mathrm{NADH} / \mathrm{NAD}^{+}$ratio, especially when slaC was knocked out. This was probably because the 2,3-BD pathway which participated in the regulation of NADH/ $\mathrm{NAD}^{+}$ratio (Celinska and Grajek 2009) was disturbed, while the synthesis of the by-products such as lactic acid, ethanol, and succinate was NADH-dependent. As shown in Table 6, the concentrations of lactic acid, ethanol, and succinate in MG12 and MG 14 were higher than that in MG1 and MG13. It could be inferred that the higher $\mathrm{NADH} / \mathrm{NAD}^{+}$ratio might result in higher by-products concentrations. This could also explain the lower comprehensive titer of AC and 2,3-BD in MG12 and MG14. Besides, a small amount of 2,3-BD was detected in the fermentation broth of MG14, which indicated that another 2,3-BD pathway might exist in $S$. marcescens MG1. 
Enhanced AC production by overexpression SlaR

$\mathrm{SlaR}$, as the transcriptional regulator of the $\mathrm{AC}$ cluster in $S$. marcescens MG1, was reported to be essential for the expression of slaA and slaB, which direct the synthesis of AC in S. marcescens MG1 (Rao et al. 2012). Since moderate expression of the transcription regulator ALsR under $\mathrm{P}_{\text {bdhA }}$ (promoter of $b d h \mathrm{~A}$ ) has been proved to efficiently increase acetoin production in Bacillus subtilis, similar procedure was attempted in Serratia marcescens MG1. In our previous work, $P_{\mathrm{C}}$ was proved to be a moderate promoter in MG1 (data not shown). $P_{\mathrm{C}}$ in MG1 might function similar to $\mathrm{P}_{\mathrm{bdhA}}$ in Bacillus subtilis. $P_{\mathrm{A}}$ and $P_{\mathrm{R}}$ were the only two related promoters in the AC operon. In order to find the most suitable promoter, experiments were carried out with these promoters.

In this study, three plasmids were constructed for sla $\mathrm{R}$ expression under the promoter of $P_{\mathrm{C}}, P_{\mathrm{A}}$, and $P_{\mathrm{R}}$, respectively. The resulting plasmids $\mathrm{pACP}_{\mathrm{C}}-$ sla, $\mathrm{pACP}_{\mathrm{A}}$-slaR, and $\mathrm{pACP}_{\mathrm{R}}$-sla $\mathrm{R}$ were transformed into MG14 by electroporation. The resulting strains were named MG15, MG16, and MG17. The integrated activity of $\alpha$-ALS and $\alpha$-ALDC in strains MG14, MG15, MG16, and MG17 was determined at approximately $30 \mathrm{~h}$ in flask fermentation (Fig. 2). The results showed that the comprehensive activity of $\alpha$-ALS and $\alpha$-ALDC was $4.79,1.05$, and $2.82 \mathrm{U} / \mathrm{mg}$ in strains MG15, MG16, and MG17, respectively, while the activity was $0.97 \mathrm{U} / \mathrm{mg}$ in strain MG14.
These results indicated that the expression of SlaR resulted in more efficient expression of sla $\mathrm{AB}$ under the control of $P_{\mathrm{C}}$ than $P_{\mathrm{A}}$ or $P_{\mathrm{R}}, 394 \%$ improvement of the integrated activity of $\alpha$-ALS and $\alpha$-ALDC was detected in MG15.

As shown in Fig. 3, cell growth, sucrose consumption rate, and $(3 R)$-AC production were determined in the engineered strains. Strain MG15 had the lowest cell density $\left(\mathrm{OD}_{600}=6.24\right)$, but the highest $(3 R)$-AC production of $27.74 \mathrm{~g} / \mathrm{l}$, which was $29.91 \%$ higher than that of MG14. No obvious difference was detected in cell density and sucrose consumption of MG16 compared to MG14, while the (3R)-AC production was improved by $7.07 \%$. Lower cell growth rate and sucrose consumption rate was observed from MG17 than MG14, and the (3R)-AC concentration of $19.08 \mathrm{~g} / \mathrm{l}$ produced by MG17 was the lowest. In summary, the highest (3R)-AC production was achieved in MG15, so $P_{C}$ was chosen as the most suitable promoter to control the expression of SlaR.

\section{Optimization of culture conditions and medium}

To further improve the (3R)-AC production of strain MG15, the fermentation conditions and medium composition were optimized. As shown in Fig. 4, the (3R)-AC production was highest at $30^{\circ} \mathrm{C}, \mathrm{pH} 7.0,20 \mathrm{ml}$ liquid volume, and $5 \%$ inoculation size. Then the above conditions were used for optimizing the medium composition.

Table 5 Intracellular NADH, NAD ${ }^{+}$and ratios of NADH/NAD ${ }^{+}$in MG1 and its derivatives

\begin{tabular}{|c|c|c|c|c|c|c|c|c|}
\hline \multirow{2}{*}{$\begin{array}{l}\text { Strains } \\
\text { Fermentation time }(\mathrm{h})\end{array}$} & \multicolumn{2}{|l|}{ MG1 } & \multicolumn{2}{|l|}{ MG12 } & \multicolumn{2}{|l|}{ MG13 } & \multicolumn{2}{|l|}{ MG14 } \\
\hline & 18 & 36 & 18 & 36 & 18 & 36 & 18 & 36 \\
\hline Intracellular NADH ( $\left.\mu \mathrm{mol} / / \mathrm{OD}_{600}\right)$ & $0.32 \pm 0.01$ & $0.25 \pm 0.01$ & $0.41 \pm 0.02$ & $0.37 \pm 0.02$ & $0.36 \pm 0.02$ & $0.33 \pm 0.01$ & $0.45 \pm 0.02$ & $0.41 \pm 0.02$ \\
\hline Intracellular NAD ${ }^{+}\left(\mu \mathrm{mol} / \mathrm{OD}_{600}\right)$ & $0.35 \pm 0.01$ & $0.28 \pm 0.01$ & $0.31 \pm 0.01$ & $0.25 \pm 0.01$ & $0.33 \pm 0.01$ & $0.28 \pm 0.01$ & $0.28 \pm 0.01$ & $0.25 \pm 0.01$ \\
\hline Ratio of $\mathrm{NADH} / \mathrm{NAD}^{+}$ & $0.91 \pm 0.01$ & $0.89 \pm 0.01$ & $1.32 \pm 0.02$ & $1.48 \pm 0.02$ & $1.09 \pm 0.02$ & $1.18 \pm 0.01$ & $1.61 \pm 0.02$ & $1.64 \pm 0.02$ \\
\hline
\end{tabular}

Table 6 Comparison of the fermentation of S. marcescens MG 1 and its derivatives

\begin{tabular}{|c|c|c|c|c|c|}
\hline Strains & MG1 & MG12 & MG13 & MG14 & MG15 \\
\hline Fermentation time (h) & 24 & 36 & 24 & 36 & 36 \\
\hline Consumed sucrose (g/l) & $89.2 \pm 0.07$ & $87.9 \pm 0.14$ & $88.7 \pm 0.15$ & $86.3 \pm 0.31$ & $96.8 \pm 0.65$ \\
\hline $\mathrm{OD}_{600}$ & $17.76 \pm 0.31$ & $11.63 \pm 0.56$ & $17.73 \pm 0.22$ & $10.97 \pm 0.42$ & $7.28 \pm 0.23$ \\
\hline$(2 \mathrm{R}, 3 \mathrm{R})-2,3-\mathrm{BD}(\mathrm{g} / \mathrm{l})$ & $0.92 \pm 0.31$ & $2.96 \pm 0.31$ & $0.61 \pm 0.31$ & $1.55 \pm 0.31$ & $1.85 \pm 0.13$ \\
\hline$(2 \mathrm{~S}, 3 \mathrm{~S})-2,3-\mathrm{BD}(\mathrm{g} / \mathrm{l})$ & $0.67 \pm 0.11$ & $N^{a}$ & $0.71 \pm 0.09$ & ND & ND \\
\hline meso-2,3-BD (g/l) & $34.01 \pm 1.21$ & $1.28 \pm 0.31$ & $31.62 \pm 0.98$ & $1.25 \pm 0.06$ & $1.52 \pm 0.05$ \\
\hline$(3 R)-A C(g / l)$ & $2.92 \pm 0.08$ & $19.79 \pm 0.21$ & $6.52 \pm 0.08$ & $21.04 \pm 0.31$ & $39.91 \pm 1.35$ \\
\hline$(3 S)-A C(g / l)$ & $0.79 \pm 0.05$ & $0.21 \pm 0.03$ & $0.63 \pm 0.06$ & ND & ND \\
\hline Lactic acid & $3.06 \pm 0.03$ & $8.05 \pm 0.06$ & $3.42 \pm 0.03$ & $8.15 \pm 0.07$ & $6.83 \pm 0.05$ \\
\hline Ethanol & $0.7 \pm 0.01$ & $1.6 \pm 0.01$ & $0.6 \pm 0.01$ & $1.8 \pm 0.01$ & $1.5 \pm 0.02$ \\
\hline Succinic acid & $1.9 \pm 0.02$ & $3.8 \pm 0.03$ & $2.1 \pm 0.02$ & $4.1 \pm 0.04$ & $2.6 \pm 0.04$ \\
\hline
\end{tabular}

a Not detected 


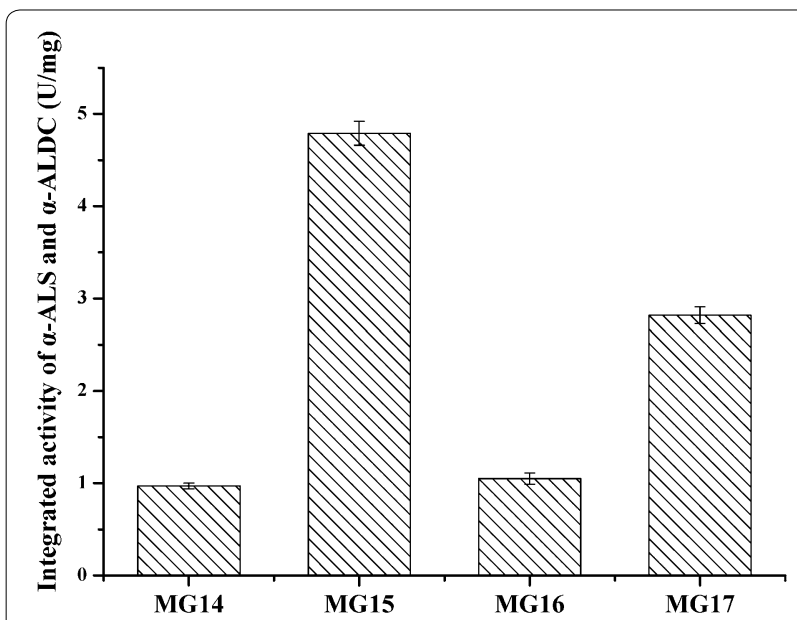

Fig. 2 The integrated activity of a-ALS and a-ALDC of MG14, MG 15, $M G 16$, and MG17

Plackett-Burman design was first used to screen significant factors from the seven variables including sucrose, yeast extract, sodium citrate, sodium acetate, $\mathrm{NH}_{4} \mathrm{H}_{2} \mathrm{PO}_{4}$, $\mathrm{MgSO}_{4}$, and $\mathrm{MnSO}_{4}$, based on preliminary experiments and related reports. The design details and the corresponding results are presented in Table 3. Analysis to the experimental data showed that there was a wide variation of $(3 R)$-AC production from 19.78 to $32.31 \mathrm{~g} / \mathrm{l}$ in the 12 experiments. After analysis of the regression coefficients, sucrose, yeast extract, sodium citrate, and $\mathrm{NH}_{4} \mathrm{H}_{2} \mathrm{PO}_{4}$ showed positive effects, while sodium acetate, $\mathrm{MgSO}_{4}$, and $\mathrm{MnSO}_{4}$ showed negative effects. The $p$ values of sucrose and sodium acetate were $>0.05$, so they were considered significant, whereas the other factors were considered insignificant. Although sucrose and sodium acetate were identified to be two significant medium components, the optimal levels of them were still need to be determined in further experiments.

Sucrose and sodium acetate were further optimized based on the results of $\mathrm{PB}$ design using CCD. The experimental design and results are shown in Table 4. After analyzing the experimental data, the following second-order polynomial equation was obtained, describing the relationship between the $(3 R)$-AC production $(Y)$ and the concentrations of sucrose $\left(X_{1}\right)$ and sodium acetate $\left(X_{2}\right)$.

$$
\begin{aligned}
Y= & -4.17+0.702 X_{1}+2.06 X_{2}-0.002744 X_{1}^{2} \\
& -0.770 X_{2}^{2}-0.0089 X_{1} X_{2}
\end{aligned}
$$

The $R^{2}$ value of this model was 0.9793 , indicating the model could explain $97.93 \%$ of the variability in the response. The adjusted $R^{2}$ was $96.45 \%$, which indicated a high significance of the model.

The three-dimensional response surface (Fig. 5) was also used to determine the effects of sucrose and sodium acetate on $(3 R)$-AC production. It was obvious that the response surface was convex in nature, suggesting that the optimum conditions were well defined. Based on the equation model and the response surface, the optimal concentration of sucrose and sodium acetate was 105 and $1 \mathrm{~g} / \mathrm{l}$, respectively; the corresponding maximum (3R)-AC production was predicted to be $39.27 \mathrm{~g} / \mathrm{l}$.

From the above results, the optimum medium composition for (3R)-AC production by $S$. marcescens MG15 was as follows (g/l): sucrose 105 , yeast extract 25 , sodium citrate 15 , sodium acetate $1, \mathrm{NH}_{4} \mathrm{H}_{2} \mathrm{PO}_{4} 0.5, \mathrm{MgSO}_{4} 0.2$, and $\mathrm{MnSO}_{4}$ 0.02. Validation experiment was carried out in triplicate test to confirm the reliability of the model equation. As shown in Fig. 6 and Table 6, the average (3R)-AC yield of $39.91 \pm 1.35 \mathrm{~g} / \mathrm{l}$ was obtained under the
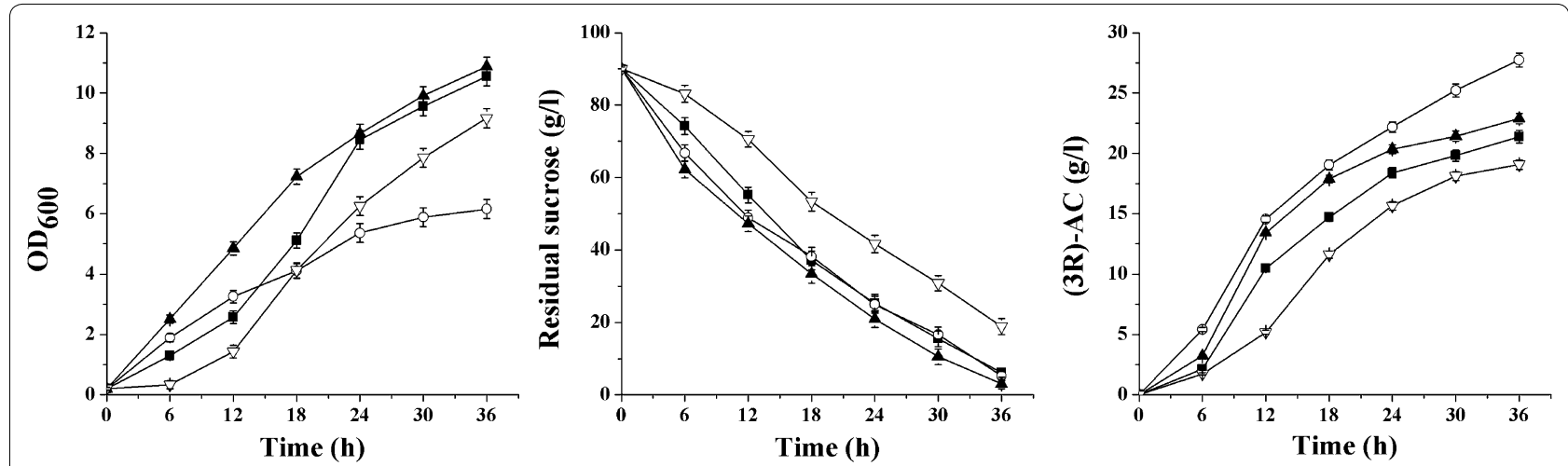

Fig. 3 Curves of cell growth, residual sucrose, and (3R)-AC production in shake flask fermentation by strain MG14 (Black square), MG15 (White circle), MG16 (Black up-pointing triangle), and MG17 (White down-pointing triangle) 

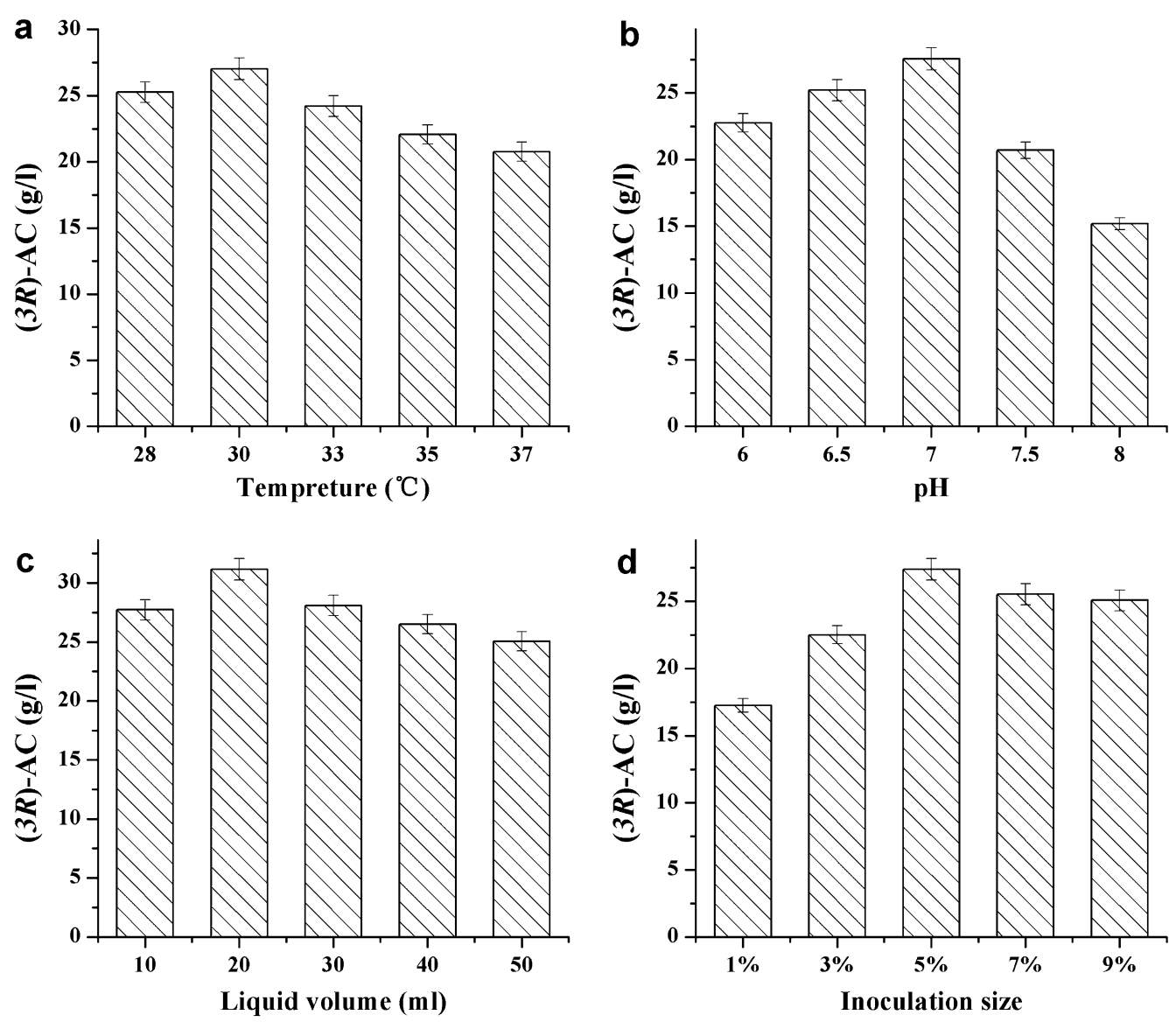

Fig. 4 Effects of different culture conditions on (3R)-AC production by Serratia marcescens MG15. a Temperature; b pH; c liquid volume; d inoculation size

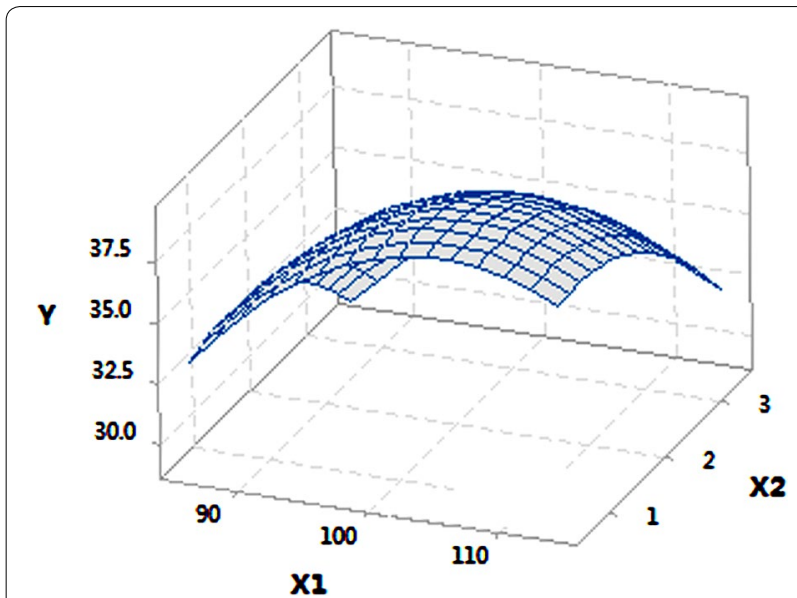

Fig. 5 Response surface figure of the mutual effects of sucrose $\left(X_{1}\right)$ and sodium acetate $\left(X_{2}\right)$ on $(3 R)$-AC production optimized condition, which was very close to the predicted maximum value of $39.27 \mathrm{~g} / \mathrm{l}$. Besides, the conversion rate was $80.13 \%$ and the productivity was $1.11 \mathrm{~g} / \mathrm{l} \mathrm{h}$. Therefore, this result indicated the validity of the model.

\section{Conclusion}

In this study, the slaC gene and $g l d \mathrm{~A}$ gene responsible for reducing $\mathrm{AC}$ to 2,3-BD were knocked out, and the resulting strain MG14 was found to produce a large amount of optically pure (3R)-AC, with a little 2,3-BD. In addition, overexpression of the transcription regulator SlaR under the control of the $\mathrm{P}_{\mathrm{C}}$ promoter was successful for enhancing (3R)-AC synthesis by $S$. marcescens MG14. Furthermore, the suitable culture conditions were optimized using single-factor experiments and the optimal medium component was developed using PB design and RSM. Based on the suitable culture conditions and the 


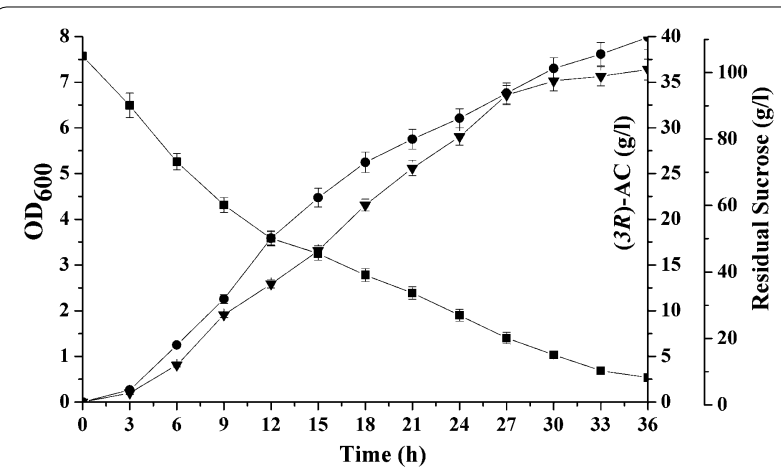

Fig. 6 Time course of (3R)-AC production of strain MG15 in the suitable culture conditions and the optimized medium. $\mathrm{OD}_{600}$ (filled up-right triangles), residual sucrose (filled squares) and (3R)-AC (filled circles)

optimized medium, the engineered S. marcescens MG16 could produce $39.91 \pm 1.35 \mathrm{~g} / \mathrm{l}(3 R)$-AC with a conversion rate of $80.13 \%$ and productivity of $1.11 \mathrm{~g} / \mathrm{l} \mathrm{h}$ in the flask fermentation.

\section{Abbreviations}

AC: acetoin; meso-2,3-BDH: meso-2,3-butanediol dehydrogenase; GDH: glycerol dehydrogenase; 2,3-BD: 2,3-butanediol; a-ALS: a-acetolactate synthase; a-ALDC: a-acetolactate decarboxylase; DA: diacetyl; AR: AC reductase; FAD: flavin adenine dinucleotide; TPP: thiamine pyrophosphate; LB: Luria-Bertani; PB: Plackett-Burman; RSM: response surface methodology; CCD: central composite design.

\section{Authors' contributions}

$X \mathrm{~L}$ wrote this manuscript. $\mathrm{LD}, \mathrm{FMB}$, and ZQW contributed general advice. YLS and LYZ edited the manuscript. All authors read and approved the final manuscript.

\section{Acknowledgements}

This work was supported by Shanghai Leading Academic Discipline Project (project B505) and National Special Fund for State Key Laboratory of Bioreactor Engineering (No. 2060204).

\section{Competing interests}

The authors declare that they have no competing interests.

Received: 20 September 2016 Revised: 9 November 2016 Accepted: 17 November 2016

Published online: 28 November 2016

\section{References}

Bai FM, Dai L, Fan JY, Truong N, Rao B, Zhang LY, Shen YL (2015) Engineered Serratia marcescens for efficient (3R)-acetoin and (2R,3R)-2,3-butanediol. J Ind Microbiol Biotechnol 42(5):779-786

Biswas R, Yamaoka M, Nakayama H, Kondo T, Yoshida K, Bisaria VS, Kondo A (2012) Enhanced production of 2,3-butanediol by engineered Bacillus subtilis. Appl Microbiol Biotechnol 94(3):651-658

Celińska E, GrajekW (2009) Biotechnological production of 2,3-butanediolcurrent state and prospects. Biotechnol Adv 27:715-725. doi:10.1016/j. biotechadv.2009.05.002

Frädrich C, March A, Fiege K, Hartmann A, Jahn D, Härtig E (2012) The transcription factor AlsR binds and regulates the promoter of the alsSD operon responsible for acetoin formation in Bacillus subtilis. J Bacteriol 194(5):1100-1112
Hao WB, Ji FL, Wang JY, Zhang Y, Wang TQ, Bao YM (2014) Biochemical Characterization of unusual meso-2,3-butanediol dehydrogenase from a strain of Bacillus subtilis. J Mol Catal B 109:184-190

Ja Sun, Zhang LY, Rao B, Han YB, Chu J, Zhu JW, Shen YL, Wei DZ (2012) Enhanced acetoin production by Serratia marcescens H32 using statistical optimization and a two-stage agitation speed control strategy. Biotechnol Bioprocess Eng 17(3):598-605

Kousoulos C, Tsatsou G, Apostolou C, Dotsikas Y, Loukas YL (2006) Development of a high-throughput method for the determination of itraconazole and its hydroxy metabolite in human plasma, employing automated liquid-liquid extraction based on 96-well format plates and LC/MS/MS. Anal Bioanal Chem 384(1):199-207

Liu YF, Zhang SL, Yong YC, Ji ZX, Ma X, Xu ZH, Chen SW (2011a) Efficient production of acetoin by the newly isolated Bacillus licheniformis strain MEL09. Process Biochem 46(1):390-394

Liu Z, Qin JY, Gao C, Hua DL, Ma CQ, Li LX, Wang Y, Xu P (2011 b) Production of (2S,3S)-2,3-butanediol and (3S)-acetoin from glucose using resting cells of Klebsiella pneumonia and Bacillus subtilis. Bioresour Technol 102(22):10741-10744

Lorenzo VD, Herrero M, Jakubzik U (1990) Mini-TnS transposon derivatives for insertion mutagenesis, promotor probing, and chromosomal insertion of cloned DNA in gram-negative Enbacteria. J Bacteriol 172(11):6568-6572

Marion MB (1976) A rapid and sensitive method for the quantitation of microgram quantities of protein utilizing the principle of protein-dye binding. Anal Biochem 72(1):248-254

Rao B, Zhang LY, Sun Ja SuG, Wei DZ, Chu J, Zhu JW, Shen YL (2012) Characterization and regulation of the 2,3-butanediol pathway in Serratia marcescens. Appl Microbiol Biotechnol 93(5):2147-2159

Renna MC, Najimudin N, Winik LR, Zahler SA (1993) Regulation of the Bacillus subtilis alsS, alsD, and alsR genes involved in post-exponential-phase production of acetoin. J Bacteriol 175(12):3863-3875

Snoep JL, Joost M, de Mattos T, Neijssel OM (1991) Effect of the energy source on the NADH/NAD ratio and on pyruvate catabolism in anaerobic chemostat cultures of Enterococcus faecalis NCTC 775. FEMS Microbiol Lett 81:63-66

Sun L, Yang F, Sun HB, Zhu TC, Li XH, Li Y, Xu ZH, Zhang YP (2015) Synthetic pathway optimization for improved 1,2,4-butanetriol production. J Ind Microbial Biotechnol 43(1):67-78. doi:10.1007/s10295-015-1693-7

Wang DX, Zhou JD, Chen C, Wei D, Shi JP, Jiang B, Liu PF, Hao J (2015) R-acetoin accumulation and dissimilation in Klebsiella pneumoniae. J Ind Microbiol Biotechnol 42(8):1105-1115

Wayne LN (2008) The Bacillus subtilis ydjL (bdhA) gene encodes acetoin reductase/2,3-butanediol dehydrogenase. Appl Environ Microb 74(22):6832-6838

Xiao ZJ, Lu JR (2014) Strategies for enhancing fermentative production of acetoin: a review. Biotechnol Adv 32(2):492-503

Xiao ZJ, Xu P (2007) Acetoin metabolism in bacteria. Crit Rev Microbiol 33(2):127-140

Xiao ZJ, Lv CJ, Gao C, Qin JY, Ma CQ, Liu Z, Liu PH, Li LX, Xu P (2010) A novel whole-cell biocatalyst with $\mathrm{NAD}^{+}$regeneration for production of chiral chemicals. PLoS ONE 5(1):e8860

Yang TH, Rathnasingh C, Lee HJ, Seung D (2014) Identification of acetoin reductases involved in 2,3-butanediol pathway in Klebsiella oxytoca. J Biotechnol 172:59-66

Zhang LY, Chen S, Xie HB, Tian YT, Hu KH (2012) Efficient acetoin production by optimization of medium components and oxygen supply control using a newly isolated Paenibacillus polymyxa CS107. J Chem Technol Biotechnol 87(11):1551-1557

Zhang X, Zhang RZ, Yang TW, Zhang J, Xu MJ, Li HZ, Xu ZH, Rao ZM (2013a) Mutation breeding of acetoin high producing Bacillus subtilis blocked in 2,3-butanediol dehydrogenase. World J Microb Biotechnol 29(10):1783-1789

Zhang X, Zhang RZ, Bao T, Yang TW, Xu MJ, Li HZ, Xu ZH, Rao ZM (2013b) Moderate expression of the transcriptional regulator ALsR enhances acetoin production by Bacillus subtilis. J Ind Microbiol Biotechnol 40(9):1067-1076

Zhang LY, Xu QM, Peng XQ, Xu BH, Wu YH, Yang YL, Sun SJ, Hu KH, Shen YL (2014) Cloning, expression and characterization of glycerol dehydrogenase involved in 2,3-butanediol formation in Serratia marcescens H30. J Ind Microbiol Biotechnol 41(9):1319-1327

Zhang LJ, Liu QY, Ge YS, Li LX, Gao C, Xu P, Ma CQ (2016) Biotechnological production of acetoin, a biobased platform chemical, from a lignocellulosic resource by metabolically engineered Enterobacter cloacae. Green Chem 18:1560-1570 